\title{
Reveal the size effect on the plasticity of ultra-small sized Ag Nanowires with in situ atomic-scale microscopy
}

Deli Kong ${ }^{a}$, Shiduo Sun ${ }^{\mathrm{a}}$, Tianjiao Xin ${ }^{\mathrm{a}}$, Lirong Xiao ${ }^{\mathrm{a}}$, Xuechao Sha ${ }^{\mathrm{a}}$, Lu Yan ${ }^{\mathrm{a}}$, Shengcheng Mao ${ }^{\mathrm{a}}$, Jin Zou ${ }^{\mathrm{b}, \mathrm{c}}$, Lihua Wang ${ }^{\mathrm{a}, *}$, Xiaodong $\operatorname{Han}^{\mathrm{a}, *}$

${ }^{a}$ Institute of Microstructure and Properties of Advanced Materials, Beijing University of Technology, Beijing, China

${ }^{\mathrm{b}}$ Materials Engineering, The University of Queensland, Brisbane, QLD 4072, Australia

${ }^{\mathrm{c}}$ Centre for Microscopy and Microanalysis, The University of Queensland, Brisbane, QLD 4072, Australia

*Corresponding authors: xdhan@ @jut.edu.cn; wlh@bjut.edu.cn;

\begin{abstract}
Revealing the atomic-scale deformation mechanisms of metallic nanowires (NWs) is important for their practical application. However, there are few reports providing direct atomic-scale experimental elucidation on those metallic NWs. Here, we conduct serial in situ deformation tests on silver (Ag) nanowires with diameters of 3-11 nm. The in situ atomic-scale observations reveal a transition in the deformation mechanism with a decrease in the diameter of Ag NWs. For the [554] and [001] oriented NWs with diameters of $\sim 11 \mathrm{~nm}$, the plastic deformation is dominated by full dislocation that involves leading and trailing partial dislocations, whereas the full or extended dislocations are rarely observed in the NWs with diameters in the range of $5-8 \mathrm{~nm}$, and their plastic deformation is governed by SF generation and annihilation.

Moreover, for the $[\overline{111}]$ oriented NWs, $60^{\circ}$ mixed and pure edge dislocations are frequently observed when the diameter is approximately $5 \mathrm{~nm}$ and the plastic deformation is accommodated by relative slip between two adjacent $\{111\}$ planes for NWs with diameters below $\sim 3 \mathrm{~nm}$. These results indicate that the plastic deformation not only depends on the size of NWs but also can be significantly impacted by the loading orientation.
\end{abstract}

Key words: silver nanowire; size effect; in situ; plasticity; atomic-scale. 


\section{Introduction}

Metallic Nanowires (NWs) have attracted a great deal of interest not only because of their potential in nanotechnology applications but also for their fundamental scientific interest $[1,2]$. However, NWs are typically used under external stress and/or strain to achieve novel properties and functions for practical applications [3, 4]. A dynamic atomic-scale elucidation of deformation mechanisms of NWs under external stress and/or strain is therefore fundamentally important for developing new materials with desired properties[1,2]. In situ nanomechanical testing is a powerful means to study the deformation mechanisms of NWs through real time imaging. In recent years, many experimental studies have been conducted to investigate the atomic-scale deformation dynamics of metallic NWs such as $\mathrm{Au}$ [5-9] and $\mathrm{Cu}[10]$. Based on these experimental observations, it has been speculated that there is a transition in the deformation model from full dislocations to partial dislocations with the decrease of the grain size $[5-8,11,12]$. Therefore, it is expected that the partial dislocations should dominate the plastic deformation in metallic NWs with diameters at the nanometer level.

There are very few direct atomic-scale experimental reports on the deformation behavior of metallic NWs with ultralow SF energy, such as Ag. The atomic-scale understanding of deformation mechanisms of Ag NWs so far has relied on previous molecular dynamics (MD) simulations[13-18]. Most of these MD simulations have predicted that the deformation is controlled by partial dislocations for the low stacking fault energy metals. MD predictions can provide important insights into the deformation mechanisms of NWs; however, the validity of these simulation results is still debatable under the experimental conditions. In addition, most of the previous studies have only considered the size effect on the deformation mechanism, although the loading orientation and stacking-fault (SF) energy also play a non-negligible role in determining the deformation mechanism. Thus, the atomic-scale observation of dynamic deformation process of $\mathrm{Ag}$ NWs under different loading orientations is necessary for understanding the deformation mechanisms of metallic NWs with ultralow SF energy.

\section{Experiment details}

In this study, we conducted a series of in-situ tensile tests on Ag NWs inside a high-resolution transmission electron microscopy (HRTEM) using an in-lab developed deformation device. The deformation mechanism of Ag nanowires with diameters in the range of 3-11 $\mathrm{nm}$ was investigated at the atomic scale in real time. Our in situ atomic-scale observations show a clear transition in deformation mode as the size of the Ag NWs decreased. However, the transition not only depends on the NWs but also can be significantly impacted by the loading orientation and the SF energy.

The Ag NWs with different diameters were obtained using our recently developed method $[19,20]$. By pulling the nanocrystalline thin film (prepared by the magnetron sputtering method), many ligaments that connect the opening cracks are formed, which are often made of a single grain with freshly opened surfaces. Each such ligament can be treated as a single-crystalline NW. The uniaxial tensile tests of 
Ag NWs were conducted inside a JEOL-2010F TEM $(200 \mathrm{kV})$ at strain rates from $\sim 10^{-3} \mathrm{~s}^{-1}$ to $\sim 10^{-4} \mathrm{~s}^{-1}$ and at temperatures (below $\sim 60^{\circ} \mathrm{C}$ ) far below the melting point of $\mathrm{Ag}$, using an in-lab developed TEM tensile device[19, 20].

\section{Results and discussion}

Figure 1 shows the atomic-resolution TEM images illustrating the tensile process of an Ag NW. The NW is [110] direction oriented and the tensile-loading direction is [554] (as indicated by the red arrow). No dislocation is observed before the elastic strain limit of the single-crystalline Ag NW with a diameter of $\sim 11 \mathrm{~nm}$, as seen in Figure 1a. A similar trend was observed for many other individual Ag NWs with diameters of $\sim 10 \mathrm{~nm}$. Figure $1 \mathrm{~b}$ is a typical HRTEM image captured during the plastic deformation showing that two dislocations, namely " 1 " and " 2 " nucleated at the top and bottom surfaces of the NW, respectively. The detailed analysis indicated that " 1 " and " 2 " are extended $60^{\circ}$ full dislocation and $60^{\circ}$ full dislocation, respectively. The extended dislocation is formed by the emission of a leading partial dislocation followed by a trailing partial dislocation on the same plane with a Burgers vector of $\mathbf{b}=\frac{1}{2}[10 \overline{1}]$, indicated by the red arrow in Figure 1b. The two partial dislocations are separated by a SF, and the width of the SF is determined by the local stress state. The inset shows the enlarged HRTEM image from the red-framed region of Figure 1b, in which the local Burgers circuit is drawn to determine the Burgers vector of dislocation " 2 "; the extra plane is seen for the (111) plane. This configuration represents a typical $60^{\circ}$ full dislocation. These nucleated extended dislocations can travel across the NWs and eventually can escape from the surface of NWs. As shown in figure 1c, the extended dislocation " 1 " escaped during further straining, and full dislocation " 2 " dissociated into an extended dislocation. Similarly, dislocation "2" also escaped with the increasing strain. Meanwhile, a full dislocation

" 3 " with $\mathbf{b}=\frac{1}{2}[101]$ and a SF resulting from a partial dislocation are observed in the $\mathrm{NW}$, as seen in figure $1 \mathrm{~d}$. This result is consistent with the previous experimental observations that the plastic deformation is controlled by the slip of partial dislocations involving leading and trailing partial dislocations.

In addition to the leading and trailing partial dislocations resulting in the full dislocation events, SF resulting from a single partial dislocation is also observed. Figure 2 presents a series of in situ HRTEM images showing the dislocation dynamics involving the nucleation of partial dislocations in a silver nanowire with a diameter of $\sim 8 \mathrm{~nm}$ along the [110] axis. No dislocation is detected in the NW before its elastic limit, as seen in Figure 2a. During continuous straining, three SFs, "1', "2", and "3", are observed due to the nucleation and glide of partial dislocations (Figure 2b). Figure 2c shows that the SF " 1 " is eliminated by a trailing partial dislocation. Another two partial dislocations are nucleated from the surface of NW upon loading and then propagated on a $\{111\}$ slip plane leaving behind two SFs ( "4" and "5" as seen in Figure 2d). The extensive elongation of the NW led to the elimination of SFs " 2 " and "4" (Figures 2d-f), which is similar to the SF "1" observed in Figure 2c. This SF 
generation and annihilation caused by the nucleation of leading and trailing partial dislocations is prevalent during the tensile test. In this study, we found that the plasticity is dominated by a leading partial dislocation nucleating first from the NW surface. After this leading partial dislocation escaped from the another surface of NW, a trailing partial dislocation is nucleated from the same location of the leading partial dislocation, thereby eliminating the SF and preventing the formation of extended dislocation (see Supplementary movies 1). This indicates that the NW size can significantly affect the plastic deformation of Ag NWs.

Figure 3 illustrates two in situ HRTEM observations of partial dislocation dynamics during tensile loading of a NW with a diameter of $6.5 \mathrm{~nm}$ along the [001] direction. Before the elastic limit, no dislocation is detected in the NW as seen in Figure 3a. During continuous straining, four SFs "1', "2", “3", and "4" are observed (Figure $3 \mathrm{~b}$ ) due to the nucleation and glide of partial dislocations in the NW. Most of the SFs are eliminated after further pulling, similar to SF "1", as observed in Figure 2c. During the deformation, the plasticity is dominated by the nucleation and propagation of the first leading partial dislocation leaving behind a SF, followed by a trailing partial dislocation eliminating the SF without forming the extended dislocation.

In addition to the size effect on the dislocation configuration, we found that the tensile-loading direction can also affect the deformation mechanisms of Ag NWs. Figures 4a-e show a series of TEM images illustrating the tensile-loading process of an $\mathrm{Ag}$ NW with a diameter of $\sim 5 \mathrm{~nm}$ along [111]. No dislocation is observed in the NW at relatively low strain rates, as seen in Figure 4a. In Figure 4b, a full dislocation " 1 " is nucleated at the upper surface of the NW. The detailed analysis indicated that the full dislocation is a $60^{\circ}$ mixed dislocation with a Burgers vector $(b)$ of $\mathbf{b}=\frac{1}{2}[101]$. The pure-edge dislocations also frequently nucleate after further pulling, as noted by the red-framed region of Figure $4 \mathrm{~b}$ and Figure $4 \mathrm{c}$. Figure $4 \mathrm{f}$ and Figure $4 \mathrm{~g}$ are enlarged HRTEM images corresponding to the red-framed regions of Figure $4 \mathrm{~b}$ and Figure $4 \mathrm{c}$, respectively. Local Burgers circuits are drawn to determine the Burgers vectors and the extra planes of atoms are seen for both the $(111)$ and the (111) planes (Figure 4e). This configuration represents a Lomer dislocation (LD)[21, 22] with $\mathbf{b}=$ $\frac{1}{2}[110]$. This is the first time in situ atomic-scale observations have revealed the formation of Lomer-Cottrell lock dislocation in $\mathrm{Ag} \mathrm{NWs}$. Interestingly, SF or extended dislocation resulting from the partial dislocations is rarely observed during tensile loading. This full dislocation dominated deformation mechanism is different from the previous theoretical and experimental studies, which predicted that the partial dislocation is the dominant plasticity in nanoscale metals with low SF energy.

The diameter of the NW decreased due to extensive tensile elongation. The plastic deformation is controlled by the discrete cooperative slip events on two conjugate $\{111\}$ planes for the NWs with diameters of $\sim 3 \mathrm{~nm}$, resulting in a number 
of enlarged steps on the surface of the NW as seen in Figure 4e. These surface steps are several atomic planes apart exhibiting a saw-tooth morphology compared to that in Figure 4d and correspond to the quantized plastic deformation (see Supplementary movies 2). The relative slip between two adjacent $\{111\}$ planes can lead to the inhomogeneous deformation, which resulted in a rapid reduction of cross-section area (Figure 4e). During the plastic deformation, a SF or extended dislocation resulting from a partial dislocation is rarely observed.

These results show that there is an obvious size effect on the plastic deformation of Ag NWs, which is schematically illustrated in Figure 5. For [554] and [001] oriented NWs with diameters above $\sim 11 \mathrm{~nm}$, the plastic deformation is governed by full or extended dislocation, which involves leading and trailing partial dislocations (Figure 5a). In contrast, the full or extended dislocation is rarely observed for the NWs with diameters in the range of $\sim 5-8 \mathrm{~nm}$, which shows the SF generation and annihilation as the dominant deformation mechanism, as illustrated in Figure $5 \mathrm{~b}$. Moreover, the loading direction can also significantly affect the deformation mechanisms of Ag NWs. The plastic deformation is dominated by the full dislocation that involves mixed and pure edge dislocations (Figure 5c) for the [111] oriented NWs with diameters above $\sim 5 \mathrm{~nm}$, whereas the plastic deformation is accommodated by the relative slip between two adjacent $\{111\}$ planes for the NWs with diameters below $\sim 3$ $\mathrm{nm}$ (Figure 5d).

The in situ observations show a distinctive size effect on the deformation mechanisms of [554] and [001] oriented Ag NWs, in which there is a transition from extended dislocations to partial dislocations. This transition is similar to the NC case, in which dislocations switch from full to partial as the grain size decreases below a critical value[19, 23]. For $\mathrm{Ag} \mathrm{NW}$ with a SF energy of $\sim 18 \mathrm{~mJ} / \mathrm{m}^{2}[24,25]$ and a shear modulus of $56.7 \mathrm{GPa}[26]$, the critical grain size is $\sim 50 \mathrm{~nm}$. By considering the NW diameter equivalent to the grain size, it is reasonable observed that the plastic deformation is governed by leading and trailing partial dislocations in these small-size NWs. In large-sized NWs, the trailing partial dislocation is always nucleated before the leading partial dislocation escaped from the surface of NW. Thus, extended or full dislocations are observed. For relatively small-sized NWs, leading partial dislocation can easily escaped from the surface of NW before the trailing partial dislocation nucleation, thereby partial dislocations are frequently observed. The observed extended and partial dislocations confirmed that the MD simulations are useful in understanding the plasticity in metallic NWs despite mild differences in the strain rate.

Additionally, the schmid factors of the full and partial dislocations for the NWs with various loading directions are also calculated. For the NWs with [554], 
full dislocations is $0.334,0.408$ and 0.272 , respectively, which are relatively smaller than the partial dislocations with maximum schmid factors of 0.386 , 0.471 and 0.314 respectively. According to this calculation, it is reasonable that the plastic deformation is governed by partial dislocations in these small-sized NWs. However, the in situ atomic-scale observation indicate that the full dislocations are prevalent even in a $\sim 5 \mathrm{~nm}$ sized Ag NW with loading direction of [111]. This observation is contradict with the schmid factor calculation and previous MD simulations prediction[15, 27-35], which may be attribute to the different surface energy and morphology of [111] oriented NWs [6]. This might need more investigation. Here, the observation of full dislocation and the plasticity transition from full dislocation to relative slip between the $\{111\}$ planes indicate that the plastic deformation can be significantly impacted not only by the size but also by the loading direction, as observed in the case of the [111] oriented Ag NWs. These observations may also be valid for the metals with similar SF energy curve to Ag such as $\mathrm{Cu}, \mathrm{Au}[26,36-41]$.

\section{Conclusion}

In conclusion, extensive in situ experiments were conducted to obtain the atomic-scale tensile processes of Ag NWs with different diameters and orientations during the tensile-loading process using HRTEM. In situ investigation has provided the following important insights into the deformation mechanisms in ultra-thin $\mathrm{Ag}$ NWs: (1) the dynamic atomic-scale plastic deformation mechanism of Ag NWs with diameters below $\sim 10 \mathrm{~nm}$, which is rarely investigated experimentally; (2) the size effect on the dislocation mechanisms in Ag NWs is different from that in metals with high SF energy; (3) the plastic deformation can be significantly impacted not only by the size but also by the SF energy and loading direction; (4) the plasticity transition from extended dislocation to the SF generation and annihilation as the diameter decreased for the [554] and [001] oriented NWs; and (5) the switching of the deformation mechanisms directly from full dislocation to relative slip between the $\{111\}$ planes for the [111] oriented NWs. These results will advance the fundamental understanding of the deformation mechanisms in metallic NWs, particularly the ultra-thin metallic NWs and will also provide useful guidelines for the design and manufacturing of novel nanometals.

\section{Acknowledge}

This study was supported by the Key Project of C-NSF (50831001), the NSF (10102001201304) and the Beijing Nova Programme (Z1511000003150142), the Beijing PXM201101420409000053 and Beijing 211 Project. Specialized Research Fund for the Doctoral Program of Higher Education of China (3C102001201301). The Project of Construction of Innovative Teams and Teacher Career Development 
for Universities and Colleges Under Beijing Municipality (IDHT20140504), Beijing Key Lab of Microstructure and Property of Advanced Material, Beijing Univeristy of Technology and the Australian Research Council (DE150101212).

\section{Reference:}

[1] L. Wang, Z. Zhang, X. Han, In situ experimental mechanics of nanomaterials at the atomic scale, NPG Asia Materials, 5 (2013) e40.

[2] T. Zhu, J. Li, Ultra-strength materials, Progress in Materials Science, 55 (2010) 710-757.

[3] X. Han, L. Kou, X. Lang, J. Xia, N. Wang, R. Qin, J. Lu, J. Xu, Z. Liao, X. Zhang, X. Shan, X. Song, J. Gao, W. Guo, D. Yu, Electronic and Mechanical Coupling in Bent ZnO Nanowires, Adv Mater, 21 (2009) 4937-4941.

[4] C.-L. Hsin, W. Mai, Y. Gu, Y. Gao, C.-T. Huang, Y. Liu, L.-J. Chen, Z.-L. Wang, Elastic Properties and Buckling of Silicon Nanowires, Advanced Materials, 20 (2008) 3919-3923.

[5] S.H. Oh, M. Legros, D. Kiener, P. Gruber, G. Dehm, In situ TEM straining of single crystal Au films on polyimide: Change of deformation mechanisms at the nanoscale, Acta Materialia, 55 (2007) 5558-5571.

[6] H. Zheng, A. Cao, C.R. Weinberger, J.Y. Huang, K. Du, J. Wang, Y. Ma, Y. Xia, S.X. Mao, Discrete plasticity in sub-10-nm-sized gold crystals, Nat Commun, 1 (2010) 144.

[7] Y. Lu, J. Song, J.Y. Huang, J. Lou, Fracture of Sub-20nm Ultrathin Gold Nanowires, Advanced Functional Materials, 21 (2011) 3982-3989.

[8] J.H. Seo, Y. Yoo, N.Y. Park, S.W. Yoon, H. Lee, S. Han, S.W. Lee, T.Y. Seong, S.C. Lee, K.B. Lee, P.R. Cha, H.S. Park, B. Kim, J.P. Ahn, Superplastic deformation of defect-free Au nanowires via coherent twin propagation, Nano Lett, 11 (2011) 3499-3502.

[9] A. Sedlmayr, E. Bitzek, D.S. Gianola, G. Richter, R. Mönig, O. Kraft, Existence of two twinning-mediated plastic deformation modes in Au nanowhiskers, Acta Materialia, 60 (2012) 3985-3993.

[10] Y. Yue, P. Liu, Z. Zhang, X. Han, E. Ma, Approaching the theoretical elastic strain limit in copper nanowires, Nano Lett, 11 (2011) 3151-3155.

[11] X.Z. Liao, Y.H. Zhao, S.G. Srinivasan, Y.T. Zhu, R.Z. Valiev, D.V. Gunderov, Deformation twinning in nanocrystalline copper at room temperature and low strain rate, Applied Physics Letters, 84 (2004) 592.

[12] Z.W. Wang, Y.B. Wang, X.Z. Liao, Y.H. Zhao, E.J. Lavernia, Y.T. Zhu, Z. Horita, T.G. Langdon, Influence of stacking fault energy on deformation mechanism and dislocation storage capacity in ultrafine-grained materials, Scripta Materialia, 60 (2009) 52-55.

[13] A. Cao, Y. Wei, Atomistic simulations of the mechanical behavior of fivefold twinned nanowires, Physical Review B, 74 (2006).

[14] B. Wu., A. Heidelberg., J.J. Boland., Microstructure-hardened Silver nanowires, Nano Lett, 6 (2006) 468-472.

[15] A.J. Cao, Y.G. Wei, S.X. Mao, Deformation mechanisms of face-centered-cubic metal nanowires with twin boundaries, Applied Physics Letters, 90 (2007) 151909.

[16] T. Zhu, J. Li, A. Samanta, H.G. Kim, S. Suresh, Interfacial plasticity governs strain rate sensitivity and ductility in nanostructured metals, Proc Natl Acad Sci U S A, 104 (2007) 3031-3036.

[17] C. Deng., F. Sansoz, Enabling ultrahigh plastic flow and work hardening in twinned gold nanowires, Nano Lett, 9 (2009). 
[18] D. Jang, X. Li, H. Gao, J.R. Greer, Deformation mechanisms in nanotwinned metal nanopillars, Nat Nanotechnol, 7 (2012) 594-601.

[19] L. Wang, X. Han, P. Liu, Y. Yue, Z. Zhang, E. Ma, In SituObservation of Dislocation Behavior in Nanometer Grains, Physical Review Letters, 105 (2010).

[20] L. Wang, J. Teng, P. Liu, A. Hirata, E. Ma, Z. Zhang, M. Chen, X. Han, Grain rotation mediated by grain boundary dislocations in nanocrystalline platinum, Nat Commun, 5 (2014) 4402.

[21] A. Bourret, J. Desseaux, A. Renault, Core structure of the Lomer dislocation in germanium and silicon, Philosophical Magazine A, 45 (1982) 1-20.

[22] R. Madec, B. Devincre, L. Kubin, T. Hoc, D. Rodney, The role of collinear interaction in dislocation-induced hardening, Science, 301 (2003).

[23] M. Chen, E. Ma, K.J. Hemker, H. Sheng, Y. Wang, X. Cheng, Deformation twinning in nanocrystalline Aluminum, SCIENCE, 300 (2003).

[24] N. Bernstein, E. Tadmor, Tight-binding calculations of stacking energies and twinnability in fcc metals, Physical Review B, 69 (2004).

[25] S. Kibey, J.B. Liu, D.D. Johnson, H. Sehitoglu, Predicting twinning stress in fcc metals: Linking twin-energy pathways to twin nucleation, Acta Materialia, 55 (2007) 6843-6851.

[26] D.H. Warner, W.A. Curtin, S. Qu, Rate dependence of crack-tip processes predicts twinning trends in f.c.c. metals, Nat Mater, 6 (2007) 876-881.

[27] H.S. Park, K. Gall, J.A. Zimmerman, Deformation of FCC nanowires by twinning and slip, Journal of the Mechanics and Physics of Solids, 54 (2006) 1862-1881.

[28] F. Tavazza, L.E. Levine, A.M. Chaka, Elongation and breaking mechanisms of gold nanowires under a wide range of tensile conditions, Journal of Applied Physics, 106 (2009) 043522.

[29] C. Ji, H.S. Park, Geometric effects on the inelastic deformation of metal nanowires, Applied Physics Letters, 89 (2006) 181916.

[30] M.R. Sorensen, M. Brandbyge, K.W. Jacobsen, Mechanical deformation of atomic-scale metallic contacts: structure and mechanisms, Physical Review B, 57 (1998).

[31] C. Deng, F. Sansoz, Near-ideal strength in gold nanowires achieved through microstructural design, ACS Nano, 3 (2009) 3001-3008.

[32] E. Rabkin, D.J. Srolovitz, Onset of plasticity in gold nanopillar compression, Nano Lett, 7 (2007).

[33] L. Sun, A.V. Krasheninnikov, T. Ahlgren, K. Nordlund, F. Banhart, Plastic Deformation of Single Nanometer-Sized Crystals, Physical Review Letters, 101 (2008).

[34] W. Liang, M. Zhou, Response of copper nanowires in dynamic tensile deformation, Proc. Inst. Mech. Eng, 218 (2004).

[35] T. Zhu, J. Li, A. Samanta, A. Leach, K. Gall, Temperature and Strain-Rate Dependence of Surface Dislocation Nucleation, Physical Review Letters, 100 (2008).

[36] E. Tadmor, A first-principles measure for the twinnability of FCC metals, Journal of the Mechanics and Physics of Solids, 52 (2004) 2507-2519.

[37] A.G. Froseth, P.M. Derlet, H. Van Swygenhoven, Grown-in twin boundaries affecting deformation mechanisms in nc-metals, Applied Physics Letters, 85 (2004) 5863.

[38] A. Stukowski, K. Albe, D. Farkas, Nanotwinned fcc metals: strengthening versus softening mechanisms, Physical Review B, 82 (2010) 224103.

[39] H. Van Swygenhoven, P.M. Derlet, A.G. Froseth, Stacking fault energies and slip in nanocrystalline metals, Nat Mater, 3 (2004) 399-403.

[40] M.J. Mehl, D.A. Papaconstantopoulos, N. Kioussis, M. Herbranson, Tight-binding study of 
stacking fault energies and the Rice criterion of ductility in the fcc metals, Physical Review B, 16 (2000).

[41] Z.H. Jin, S.T. Dunham, H. Gleiter, H. Hahn, P. Gumbsch, A universal scaling of planar fault energy barriers in face-centered cubic metals, Scripta Materialia, 64 (2011) 605-608. 


\section{Figure captions:}

Figure 1. In situ atomic-scale observations of the extended dislocations resulting from leading and trailing partial dislocations for the [554] oriented NWs: (a) HRTEM image of an Ag NW with a diameter of $\sim 11 \mathrm{~nm}$ with no dislocation detected before the elastic limit; (b) A typical HRTEM image that captured the plastic deformation and the nucleation of two dislocations. The inset is an enlarged HRTEM image corresponding to the red-framed region; (c) The extended dislocation " 1 " escaped and full dislocation " 2 " dissociated into an extended dislocation; and (d) Similarly, dislocation " 2 " also escaped with increasing strain and a third full dislocation "3" along with a SF resulting from a partial dislocation are observed.

Figure 2. Sequential in situ HRTEM images showing the partial dislocations dynamics for the [554] oriented Ag NW with a diameter of $\sim 8 \mathrm{~nm}$ : (a) Before the elastic limit, no dislocation is detected in the NW; (b) With continuous straining, three SFs resulting from the partial dislocations are observed; (c) SF "1" is eliminated by a trailing partial dislocation emission; (d) Another two partial dislocations are nucleated from the surface of NW (viz., "4" and " 5 ") after further pulling; and (d-f) SFs " 2 " and "4" are eliminated similar to SF "1" (c).

Figure 3. In situ observation of partial dislocation dynamics during tensile loading of the [001] oriented Ag NWs: (a) Before the elastic limit, no dislocation is detected; (b) Upon further straining, four SFs "1', "2", "3", and "4" resulting from the nucleation of partial dislocations are observed.

Figure 4. A series of TEM images shows the dislocation behavior of an Ag NW with a $5 \mathrm{~nm}$ diameter along the loading direction of [111]: (a) No dislocation is detected in the NW at relatively low strain; (b) A $60^{\circ}$ full dislocation " 1 " with $\mathbf{b}=\frac{1}{2}$ [101]nucleated at the upper surface of the NW; (c, d) Nucleation of pure-edge dislocations is frequently observed as noted by the red-framed region; (f, g) Enlarged HRTEM images corresponding to the red-framed region of $(b, c)$ with a Burgers circuit to identify the dislocation representing a Lomer dislocation with $\mathbf{b}=\frac{1}{2}[110]$; and (e) For the NWs with the diameters of $\sim 3 \mathrm{~nm}$, the discrete cooperative slip on two conjugate $\{111\}$ planes led to a number of enlarged steps on the surface of the NWs.

Figure 5 is a schematic diagram showing the deformation mechanism of $\mathrm{Ag}$ NWs: (a-b) In the case of [554] or [001]oriented NWs, the plastic deformation is governed by full or extended dislocations for the NWs with diameters of $\sim 11 \mathrm{~nm}$; the SF generation and annihilation dominated the plastic deformation for the NWs with diameters in the range of $\sim 5-8 \mathrm{~nm}$; and (c-d) In the case of [111] oriented NWs, the plastic deformation is dominated by full dislocation when the diameter of NW is 
above $\sim 5 \mathrm{~nm}$; the plastic deformation is accommodated by relative slip between two adjacent $\{111\}$ planes for the NWs with diameters below $\sim 3 \mathrm{~nm}$. 


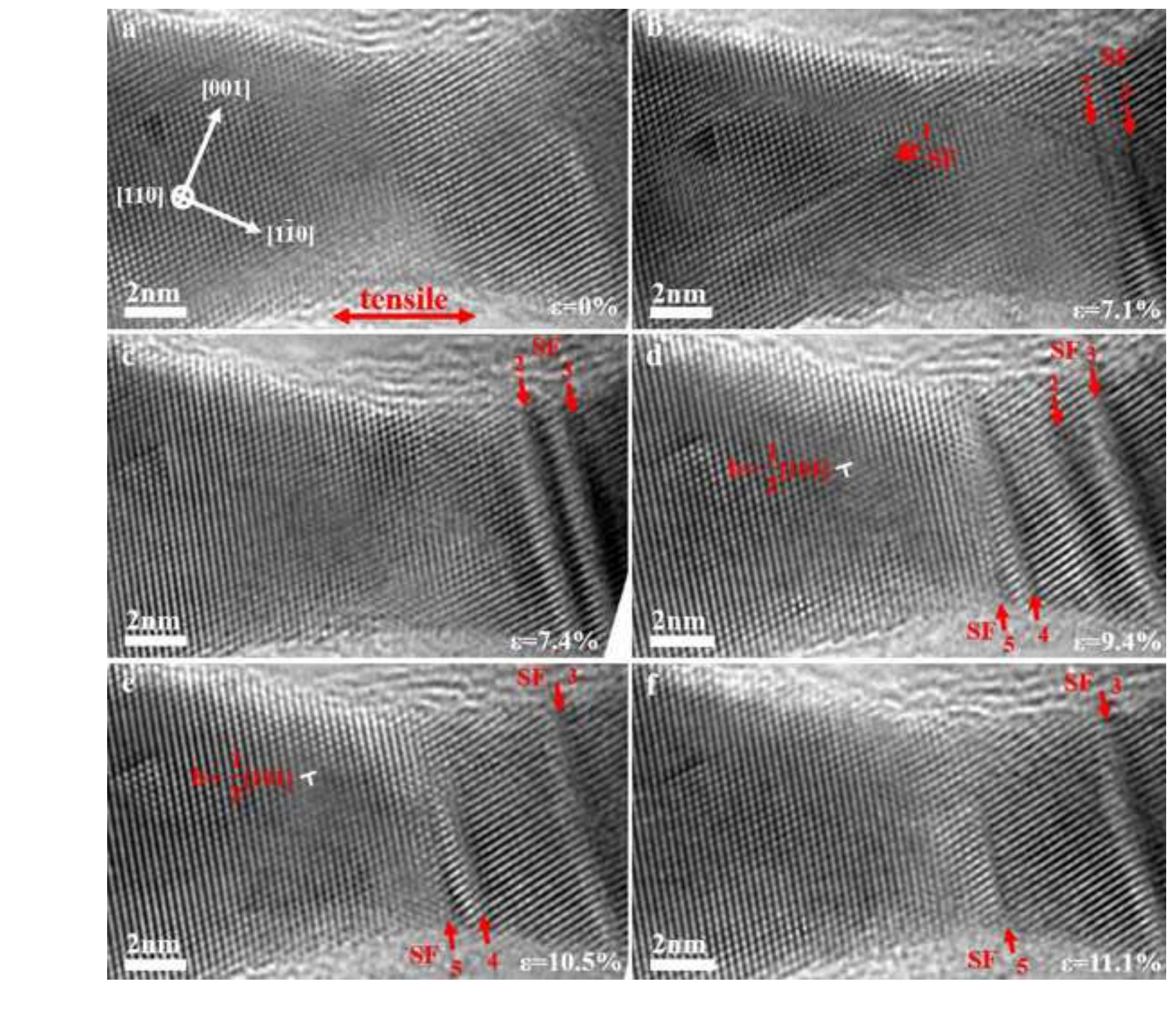




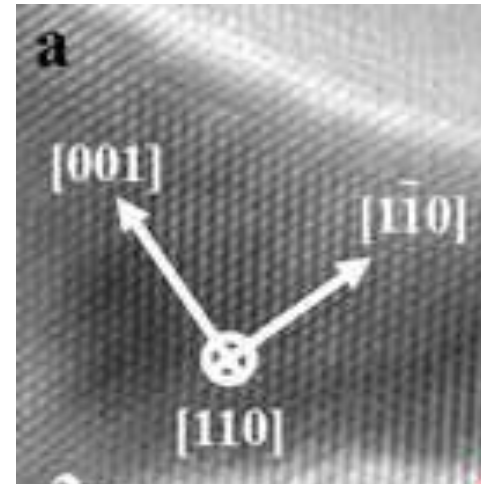

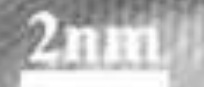

\section{$\stackrel{\text { tensile }}{\longrightarrow}$}
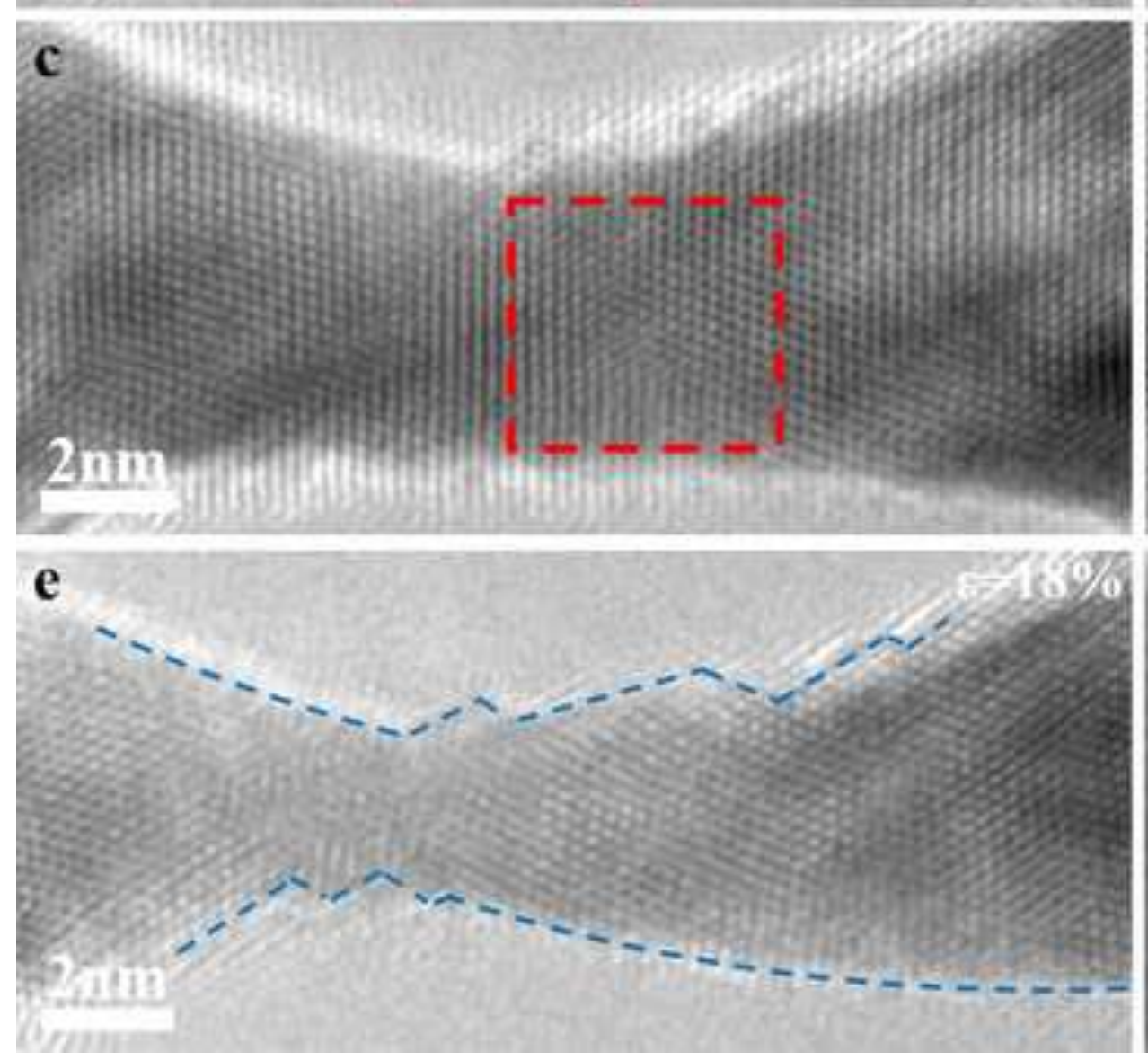
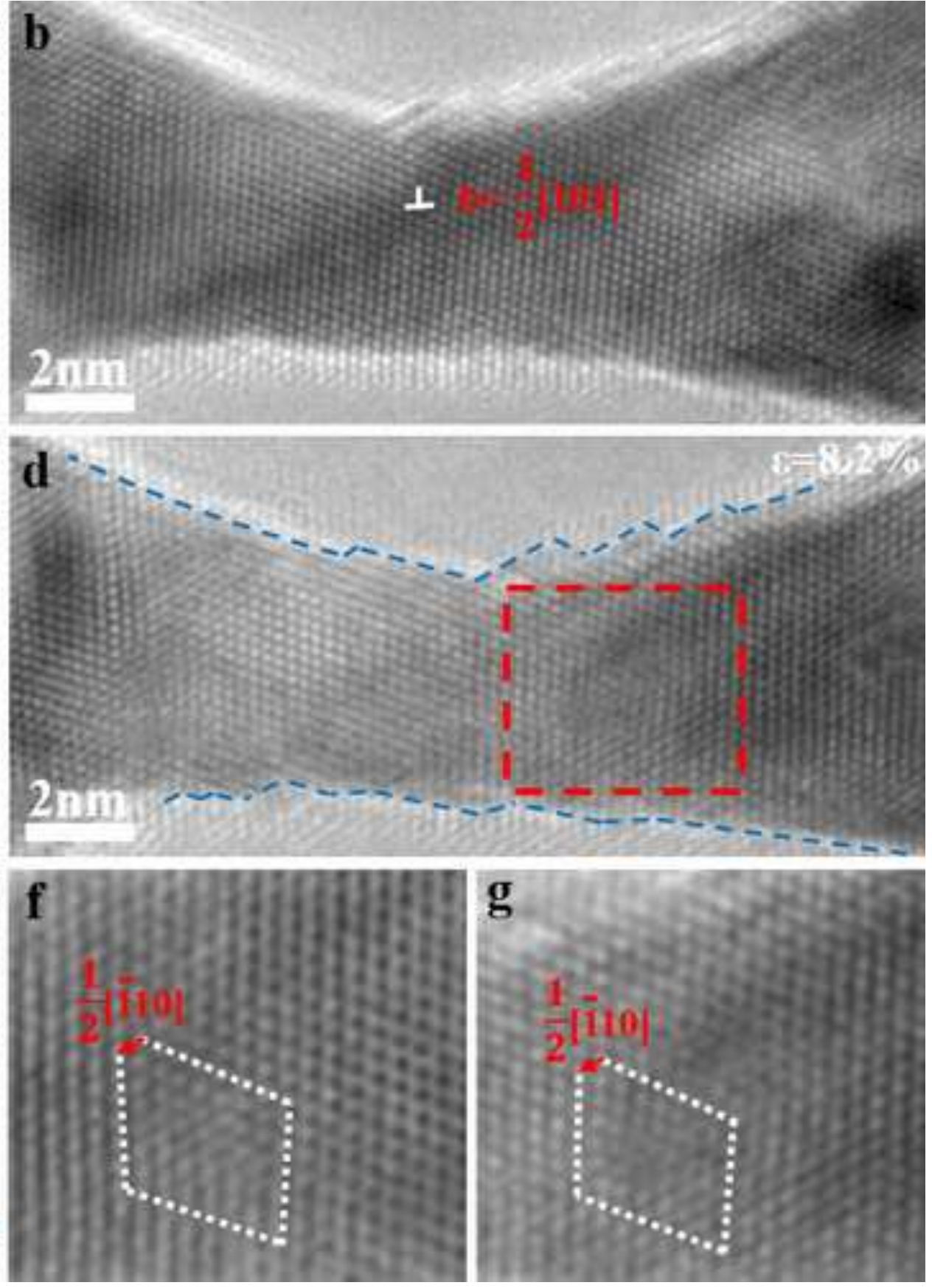

g $\frac{1}{2}[110 \mid$

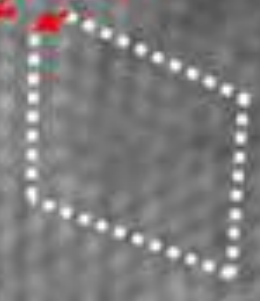



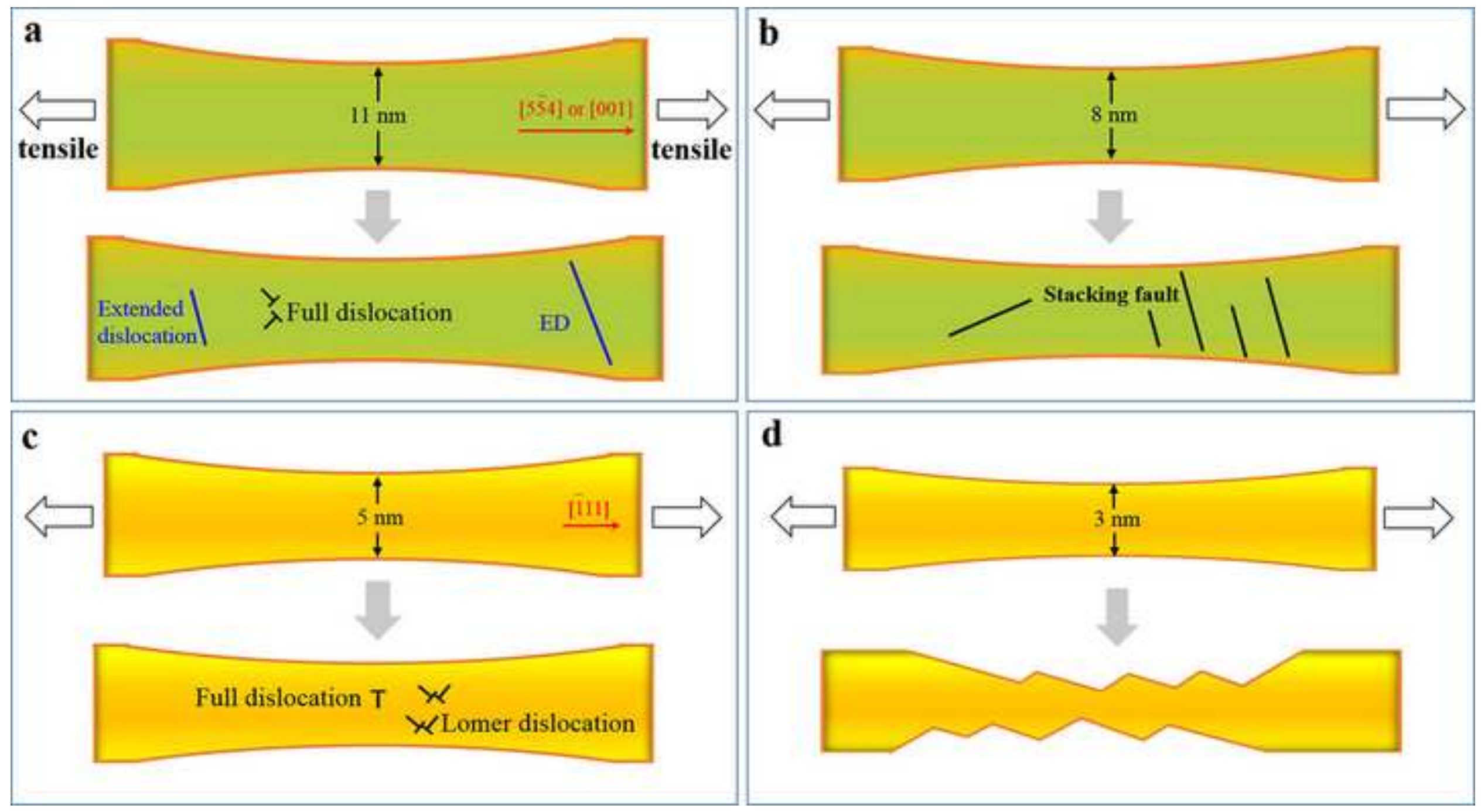